Revista Médica Sinergia

Vol. 7, Núm. 2, febrero 2022, e761 https://doi.org/10.31434/rms.v7i2.761

revistamedicasinergia@gmail.com

\title{
Fenómeno de Lucio: reporte de caso
}

\section{Lucio's phenomenon: a case report}

\author{
${ }^{1}$ Dr. Nelson Aguilar Aguilar \\ Hospital San Juan de Dios, San José, Costa Rica \\ (Di) https://orcid.org/0000-0001-7623-8339 \\ ${ }^{2}$ Dr. Giovanni Sandí Salazar \\ Hospital Rafael Ángel Calderón Guardia, San José, Costa Rica \\ (iD https://orcid.org/0000-0002-3395-0729
}

${ }^{3}$ Dra. Priscilla Muñoz Mora Investigadora independiente, San José, Costa Rica

(iD) https://orcid.org/0000-0003-4213-6275

Recibido 02/12/2021
Corregido

$25 / 12 / 2021$
Aceptado

$10 / 01 / 2022$

\section{RESUMEN}

Se presenta el caso de un paciente masculino de 25 años de edad, en quien se diagnostica Enfermedad de Hansen por manifestaciones de Fenómeno de Lucio. Debido a la poca frecuencia de presentación en nuestro medio de este fenómeno, se realiza reporte de caso para mayor conocimiento de la comunidad médica.

PALABRAS CLAVE: fenómeno de Lucio; leprorreación; Hansen.

\section{ABSTRACT}

The case of a 25 years old male patient is presented in whom Hansen Disease is diagnosed due to manifestations of Lucio's Phenomenon. Due to de infrecuency of presentation of this phenomenon in our environment, a case report is made to better understand the medical community.

KEYWORDS: Lucio's phenomenon; leprosy; Hansen.

\footnotetext{
${ }^{1}$ Médico especialista en dermatología, graduado del Instituto de Dermatología Prof. Rubem D. Azulay - CESANTA. Río de Janeiro, Brasil. Cód. MED6343. Correo: naguilarmd@yahoo.com

${ }^{2}$ Médico especialista en medicina interna y dermatología, graduado de la Universidad de Costa Rica (UCR). Cod. MED9399. Correo: Sandigiova@gmail.com

${ }^{3}$ Médica residente, graduada de la Universidad de Costa Rica (UCR). Cod. MED14542. Correo: Priscilamm24@gmail.com
} 


\section{INTRODUCCIÓN}

La lepra es una enfermedad infecciosa crónica de progresión lenta producida por el Mycobacterium leprae y Mycobacterium tuberculosis que afecta principalmente los nervios periféricos y a la piel. Se han descrito reacciones lepromatosas las cuales corresponden a estados de la enfermedad mediados inmunológicamente que alteran el curso crónico habitual y la estabilidad clínica de los pacientes con lepra. Una de las variantes es el Fenómeno de Lucio, el cual es una forma grave de leproreacción, observada principalmente en la lepra lepromatosa difusa, caracterizada por lesiones necróticas por vasculitis.

\section{DESCRIPCIÓN DEL CASO}

Se trata de un paciente masculino de 25 años quien se atiende en el servicio de dermatología del Hospital San Juan de Dios de la Caja Costarricense de Seguro Social (CCSS) en San José, Costa Rica, por úlceras en ambos miembros inferiores de aproximadamente 3 meses de evolución.

El paciente, a los 21 años de edad presentó máculas hipocrómicas a nivel de espalda, un año de evolución de madarosis y además se observa infiltración difusa en piel.

La historia clínica revela que no tiene antecedentes personales patológicos (niega alergia a medicamentos, tabaquismo, etilismo o transfusiones). Además, refiere como antecedentes quirúrgicos, una hernioplastia umbilical. No tiene antecedentes heredofamiliares de patologías cutáneas. Al examen físico se documenta un fototipo fitzpatrick IV, piel de aspecto infiltrado brillante, turgente a nivel facial y en dorso de las manos (ver figura 1.); a nivel de miembros inferiores en región pretibial y región posterior de piernas (así como también a nivel de miembros superiores en antebrazos, presenta ulceras con escara de bordes con formas abigarradas (ver figura 2.), bien definidos, angulados, algunas de forma estrellada, asociadas a cambios de hiperpigmentación e hipopigmentación y xerosis. A nivel de tórax anterior y posterior piel de aspecto turgente. No se documentó engrosamiento neural, ni anomalías sensitivas. Los laboratorios reportan:

- Baciloscopia con un índice bacilar de 3,75 .

- Hemoglobina $12.1 \mathrm{~g} / \mathrm{dL}$, leucocitos en 5700 , con $59 \%$ segmentados, linfocitos $30 \%$, plaquetas en 204.000 .

- Nitrógeno ureico $13 \mathrm{mg} / \mathrm{dL}$, creatinina en $0.66 \mathrm{mg} / \mathrm{dL}$, un aclaramiento de creatinina $156 \mathrm{ml} / \mathrm{min}$, microproteínas en orina de 24 horas en $224 \mathrm{mg}$.

- Bilirrubina total $0.4 \mathrm{mg} / \mathrm{dL}$, AST $22 \mathrm{UI} / \mathrm{L}$, ALT $21 \mathrm{UI} / \mathrm{L}, \mathrm{FA} 103 \mathrm{UI} / \mathrm{L}, \mathrm{DHL} 167$ UI/L, GGT $31 \mathrm{UI} / \mathrm{L}$.

- No estaba disponible la lepromina para realizarla en el paciente.

La biopsia del paciente presenta la piel con infiltrado inflamatorio dérmico de tipo nodular, dado por la presencia de abundantes histiocitos de citoplasma claro. Este infiltrado sigue vasos sanguíneos, nervios y anexos cutáneos (ver figura 3.).

Superficialmente hay necrosis fibrinoide de un vaso y ulceración de la epidermis. Se aprecian numerosos bacilos alcohol ácido resistentes.

\section{DISCUSIÒN}

La enfermedad de Hansen es una entidad infecciosa crónica causada por Mycobacterium leprae y Mycobacterium tuberculosis, transmitida por inhalación de bacilos después de contactos cercanos y frecuentes con pacientes no tratados, al 
Figura 1. Madarosis, piel turgente infiltrada

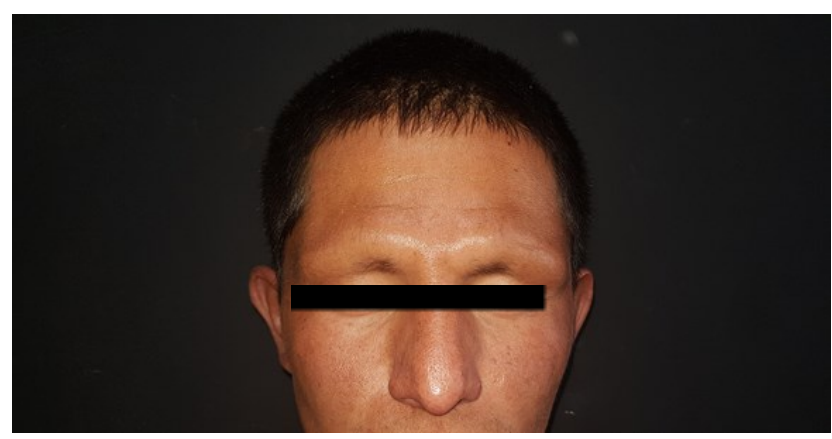

Figura 2. Ulceras con escaras necróticas de bordes angulado

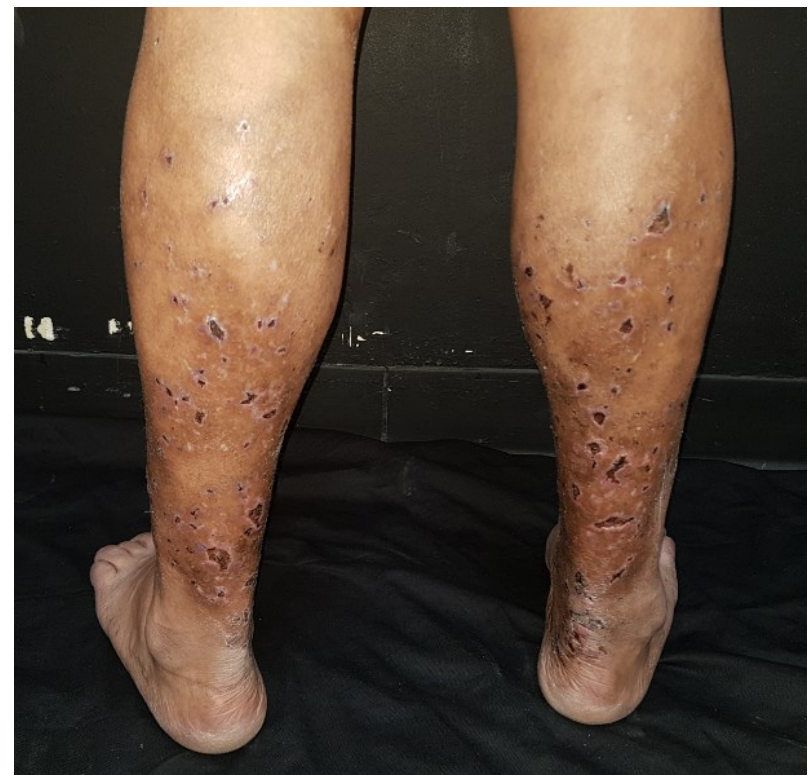

Fuente: Fotografías cortesía de Dr. Pablo Zuñiga Especialista en Patología. Hospital San Juan de Dios (CCSS)

Figura 3. Biopsia del paciente con fenómeno de Lucio

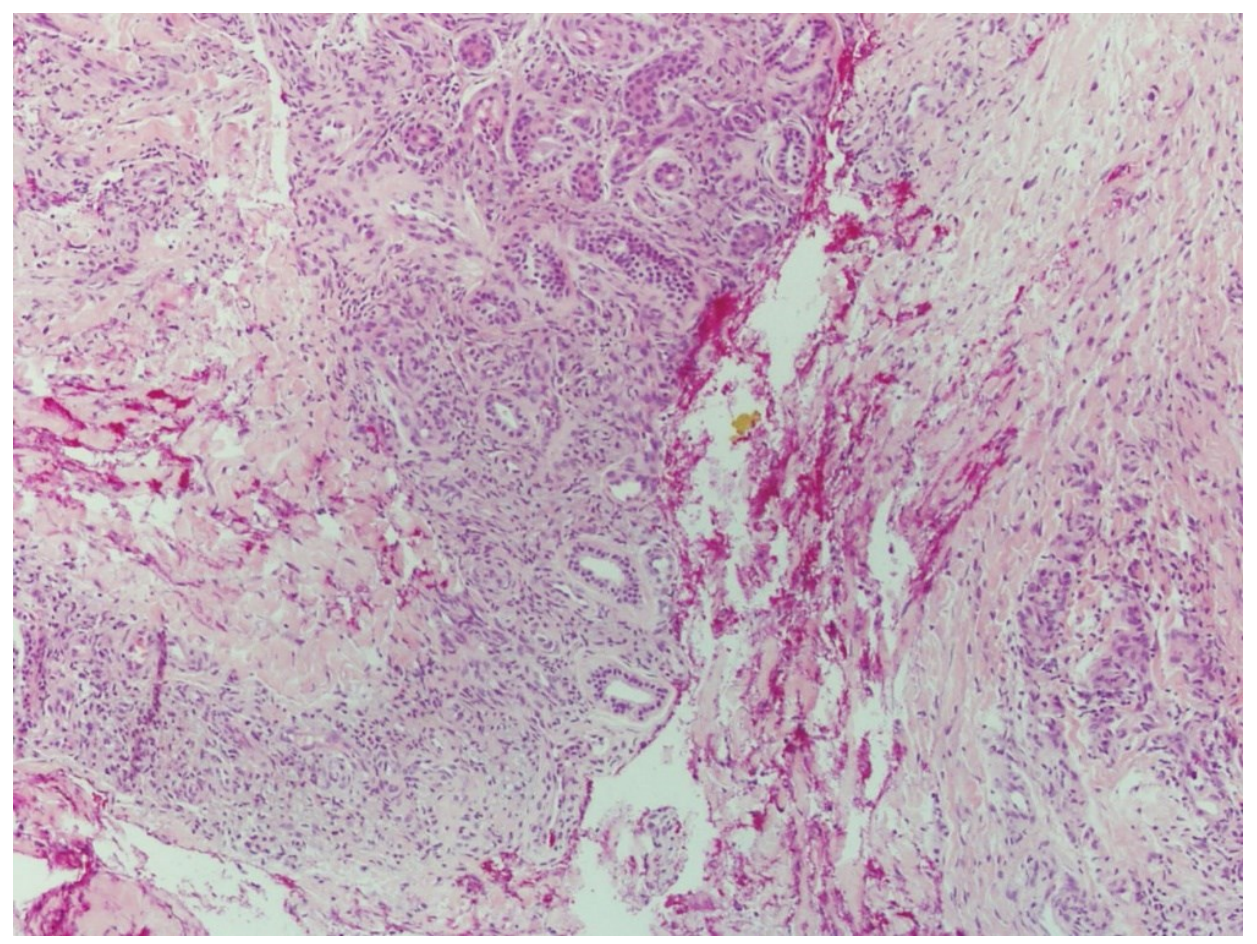

Fuente: Fotografías cortesía de Dr. Pablo Zuñiga Especialista en Patología. Hospital San Juan de Dios (CCSS) 
igual que por contacto con piel no indemne $(1,2)$. La presentación clínica de la lepra varía ampliamente y depende de la respuesta inmunológica del individuo a la infección $(3,4)$. Esta patología puede complicarse por reacciones lepromatosas que alteran el curso crónico habitual y la estabilidad clínica de los pacientes (5). Pueden separarse clínica e histopatológicamente en tres variantes diferentes: reacción inversa (tipo I), eritema nodoso (tipo II) y fenómeno de Lucio (6).

El fenómeno de Lucio es una reacción que se presenta en los pacientes con lepra lepromatosa difusa, esta leprorreacción se describió en 1853 por Lucio $(7,8)$, se presenta en algunos lugares con mayor frecuencia como México, sin embargo, se ha descrito en lugares como Costa Rica, Brasil, Argentina y la India (9). Se caracteriza por ser una vasculitis necrosante de pequeños vasos de la dermis $(10,11)$.

Clínicamente se presenta al inicio en miembros inferiores con lesiones eritematopurpúricas que con el paso de los días aparecen escaras sobre dichas lesiones y se ulceran con formas abigarradas, anguladas, de bordes netos que curan con cicatriz atrófica, generalmente los pacientes aquejan sensación dolorosa o ardorosa $(12,13)$. Las lesiones pueden extenderse a miembros superiores y a nivel facial (14). Generalmente, el diagnóstico solo se realiza después de la aparición de lesiones ulceradas, 0 incluso solo se establece después del examen anatomopatológico (15).

En ocasiones hay asociación de síntomas sistémicos como fiebre, malestar general, linfadenopatía, afección del tracto respiratorio superior que resulta epistaxis y destrucción de hueso nasal, hepatoesplenomegalia tal como se documenta en ultrasonido de abdomen realizado a nuestro paciente donde se documentó hígado aumentado de tamaño, lóbulo derecho de $183 \mathrm{~mm}$, de forma y estructura normal. Vía biliar sin hallazgos patológicos. Bazo aumentado de tamaño, $218 \mathrm{~mm}$ de longitud. La patogénesis del fenómeno de Lucio es poco comprendida hasta el momento. (16).

Se realiza biopsia cutánea de las lesiones como complemento para el diagnóstico, por lo tanto, en la histopatología se pueden observar bacilos alcohol ácido resistentes en agregados en el endotelio vascular, áreas de necrosis fibrinoide, vasculitis leucocitoclástica y necrosis isquémica epidérmica $(13,17)$. Cabe destacar que el fenómeno de lucio puede no ser fácilmente reconocido, especialmente en áreas no endémicas, lo que conduce a un diagnóstico confuso que conlleva a inicio tardío de tratamiento (18). En este caso hay diagnósticos diferenciales que se deben de tomar en cuenta tales como: vasculitis leucocitoclástica, calcifilaxis, pioderma gangrenoso, dermatitis facticia (19). La gravedad de esta patología está relacionada con el inicio tardío de la terapia que progresa hasta la muerte debido a discrasia sanguínea o sepsis (11).

El tratamiento contra el fenómeno de Lucio incluye el tratamiento multibacilar, además el uso de corticoesteroides, cuyo uso es controversial. En algunos casos se puede agregar la talidomida cuando la evolución es tórpida (20).

\section{CONCLUSIONES}

El Fenómeno de lucio debe ser valorado como diagnóstico diferencial en pacientes con enfermedad de Hansen que se presenten con lesiones ulcerosas necróticas en miembros inferiores, sin embargo, también debe considerarse en los pacientes 
sin tener el antecedente de Hansen, ya que puede ser la forma de presentación clínica, como fue el caso de nuestro paciente. Los Hallazgos histopatológicos son clave diagnóstica para poder realizar la correlación clínica-patológica de esta presentación de la enfermedad.

\section{REFERENCIAS}

1. Rocha RH, Emerich PS, Diniz LM, Oliveira MB, Cabral AN, Amaral AC. Lucio's phenomenon: exuberant case report and review of Brazilian cases. An Bras Dermatol. 2016 Sep-Oct;91(5 suppl 1):60-63

2. Fischer M. Leprosy e an overview of clinical features, diagnosis, and treatment. Journal of the German Society of Dermatology. 2017;15:801827

3. Maymone, M. B. C., Laughter, M., Venkatesh, S., Dacso, M. M., Rao, P. N., Stryjewska, B. M., Dunnick, C. A. Leprosy: Clinical Aspects and Diagnostic Techniques. Journal of the American Academy of Dermatology 2020 Jul;83(1):1-14

4. Jurado F, Rodriguez O, Novales J, Navarrete G, Rodriguez M. Lucio's leprosy: a clinical and therapeutic challenge. Clinics in Dermatolog. 2015;33:66-78

5. Ramal, C., Casapia, M., Marin, J., Celis, J. C., Baldeon, J., Vilcarromero, S., ... Legua, P. (2017). Diffuse Multibacillary Leprosy of Lucio and Latapí with Lucio's Phenomenon, Peru. Emerging Infectious Diseases, 2017 Nov;23(11):1929-1930

6. Curi PF, Villaroel JS, Migliore N, Albertengo A, Aquino ML, Ceccato F, Paira S. Lucio's phenomenon: report of five cases. Clin Rheumatol. 2016 May;35(5):1397-401

7. Saul A, Peniche A. En: Amado Saúl. Saúl Lecciones de Dermatología. $16^{a}$ edición. México: Editorial Mc Graw Hill Education; 2015. Pág. 5690.

8. Maymone MBC, Venkatesh S, Laughter M, Abdat R, Hugh J, Dacso MM, Rao PN, Stryjewska BM, Dunnick CA, Dellavalle RP. Leprosy: Treatment and management of complications. Journal of the American Academy of Dermatology. 2020 Jul;83(1):17-30.

9. Marquez C , Jurado F , Navarrete G , Rodriguez, M Lepromatous leprosy in type 2 lepra reaction. (2012). Journal of the American Academy of Dermatology, 66(4)
10. Bernardes Filho Fred, Pess Daiana, Akabane Andressa Lumi, Foss Norma Tiraboschi, Frade Marco Andrey Cipriani.Lucio's phenomenon: a life- threatening medical emergency.International Journal of Infectious Diseases (2018)

11. Rocha RH, Emerich PS, Diniz LM, Oliveira MB, Cabral AN, Amaral AC. Lucio's phenomenon: exuberant case report and review of Brazilian cases. An Bras Dermatol. 2016 Sep-Oct;91(5 suppl 1):60-63

12. Fischer M. Leprosy e an overview of clinical features, diagnosis, and treatment. Journal of the German Society of Dermatology. 2017;15:801827

13. Oliveira G, Wan Del Rey M, Hanseníase. En: Azulay L, Hanauer L, Leal F, Azulay D, Bonalumi $A$, editores Jefes. Atlas de Dermatología Da Semiologia Ao Diagnóstico. $2^{\mathrm{a}}$ Edición. Brasil: Editora Elsevier; 2013. Pág.417-423

14. Velarde-Félix, J. S., Alvarado-Villa, G., \& VeraCabrera, L. (2016). "Lucio's Phenomenon" Associated with Mycobacterium lepromatosis. The American journal of tropical medicine and hygiene, 94(3), 483-484.

15. Abulafia,L, Spinelli, L, Revendo a Hanseníase de Lucio e o Fenômeno de Lucio .Med Cutan Iber Lat Am 2005;33(3)125-133

16. Ramal, C., Casapia, M., Marin, J., Celis, J. C., Baldeon, J., Vilcarromero, S., Legua, P. (2017). Diffuse Multibacillary Leprosy of Lucio and Latapí with Lucio's Phenomenon, Peru. Emerging Infectious Diseases, 2017 Nov;23(11):1929-1930.

17. Alemu Belachew, W., \& Naafs, B. Position statement: LEPROSY : Diagnosis, treatment and follow-up. Journal of the European Academy of Dermatology and Venereology. 2019 Jul;33(7)

18. Ya SNC, Muhamad $R$, Zakaria $R$, Ishak A, Abdullah WNHW. Lucio Phenomenon: Sequelae of Neglected Leprosy. Korean Journal of Family Medicine. 2021 May;42(3):245-249

19. Naaz F, Mohanty PS, Bansal AK, Kumar D, Gupta UD. Chal- lenges beyond elimination in leprosy. Int J Mycobacteriol. 2017;6:222-228.

20. Moscella SL, Garcia-Albea V. 18 September 2016, posting date. Differencial diagnosis of leprosy , chapter 2.3. In Scollard DM, Gillis TP (ed), International textbook of leprosy.

21. Pulido A, Suarez R. Terapéutica Dermatológica: Tratamiento de las leprorreacciones. Piel. 2015; 30 (10): 681- 686 